\title{
On the wake effect in wind farm power forecasting: a new data-driven approach
}

\author{
Fabio Famoso ${ }^{1,}$, Sebastian Brusca ${ }^{1}$, Antonio Galvagno ${ }^{1}$, Michele Messina ${ }^{2}$, and Rosario \\ Lanzafame $^{2}$ \\ ${ }^{1}$ University of Messina, C.da Di Dio, Messina, 98166, Italy \\ ${ }^{2}$ University of Catania, V.le A. Doria 6, Catania 95125, Italy
}

\begin{abstract}
Wind power generation differs from other energy sources, such as thermal, solar or hydro, due to the inherent stochastic nature of wind. For this reason wind power forecasting, especially for wind farms, is a complex task that cannot be accurately solved with traditional statistical methods or needs large computational systems if physical models are used. Recently, the so-called learning approaches are considered a good compromise among the previous methods since they are able to integrate physical phenomena such as wake effects without presenting heavy computational loads. The present work deals with an innovative method to forecast wind power generation in a wind farm with a combination of GISbased methods, neural network approach and a wake physical model. This innovative method was tested with a wind farm located in Sicily (Italy), used as a case study. It consists of 30 identical wind turbines $(850 \mathrm{~kW}$ each one), located at different heights, for an overall Power peak of $25 \mathrm{MW}$. The time series dataset consists of one year with a sampling time of 10 minutes considering wind speeds and wind directions. The output of this innovative model leaded to good results, especially for medium-term overall energy production forecast for the case study.
\end{abstract}

Keywords: wind power, neural networks, GIS, wake modelling.

\section{Introduction}

Renewable energies are a key element of the modern sustainable development. They play a key role in contributing to the reduction of the impact of fossil sources and to the energy supply in remote areas where the electrical grid cannot be reached. For this reason, the renewable energy industry has grown increasingly and is expected to increase up to 2.7 times between 2010 and 2035 [1].

Renewable technologies, such as solar energy [2], biomass energy conversion systems [3-5], and especially wind power plants [6], are usually considered to be less efficient than traditional conversion systems because of the inherent random nature of the primary renewable source. This implies some difficulties to forecast energy production of such complex systems. Among them, onshore and offshore wind farms deserve a special

\footnotetext{
* Corresponding author: ffamoso@unict.it
} 
attention. The increase of installed wind farms around the world has encouraged the scientific community to better assess forecast methods for the wind energy production [7]. Traditional energy conversion systems, despite the fact are intermittent, may be considered dispatchable since the primary energy source follows a deterministic approach and the grade of uncertainty for the energy production estimation is mainly due to the random faults of several system components. On the contrary, wind generation systems are usually described as non-dispatchable systems due to the nature of the primary source, the wind power, that is usually difficult to forecast with traditional deterministic approaches. Several methods have been developed in wind farm energy production forecast. In statistical methods, such as autoregressive moving mean models (ARMA) or autoregressive integrated moving mean models (ARIMA) a vast amount of data is analyzed and they usually give proper results in the estimation of mean monthly or even higher temporal scale wind power [8]. Physical models, such as Computational fluid dynamics (CFD) based models [9], generally use global databases of meteorological measurements or atmospheric models, but they require large computational systems in order to achieve accurate results [10]. Recently, the so-called learning approaches, or AI data-driven models, are considered a good compromise among the previous groups. They may be defined as "gray box" methods since they are able to explain physical phenomena such as wake effects $[6,11]$ or faults despite the fact they do not involve any physical model. The present work deals with the application of an innovative method for medium-term forecast energy production in wind farms (weekly energy production) based on the combination of a GIS-based wake model and the application of neural networks. The rest of the paper is organized as follows: Section 2 describe the proposed methodology, wake model and the application of the neural network, Section 3 describes a wind farm used a test case, Section 4 shows model results and Section 4 is about the conclusions.

\section{Case study: A Wind farm located in Sicily (Italy)}

In this paper, in order to test and accurately verify model's results a real case of a wind farm was investigated. A wind farm located in the south of Italy, in Sicily, was analyzed and tested as case study.

The analyzed power plant is a grid-connected wind farm, constituted of 30 identical wind turbines and 3 anemometric towers, implemented by a private company in 2005 . It occupies an area of about $30 \mathrm{~km} 2$ located in the south-east of Sicily, in a rural part of the municipality of Vizzini. This areas is almost flat, with all the turbines located in a range of 600-670 meters a.s.1 without significant obstacles nearby (mountains, buildings etc.). All wind farm's turbines installed are Vestas V52 upwind type with a Ppeak of $850 \mathrm{kWp}$ and equipped with three blades. The main characteristics of this case study are presented in Table 1. 
Table 1. Main characteristics of the wind farm and the wind turbine model.

\begin{tabular}{cc}
\hline Geographical coordinates & $37^{\circ} 12^{\prime} 19.35^{\prime}{ }^{\prime} \mathrm{N}^{\circ} 4^{\circ} 44^{\prime} 12.27^{\prime}{ }^{\prime} \mathrm{E}$ \\
Overall $\mathrm{P}_{\text {peak }}$ & $25.5 \mathrm{MW}_{\mathrm{p}}$ \\
$\mathrm{N}^{\circ}$ wind turbines & 30 \\
$\mathrm{~N}^{\circ}$ anemometric towers & 3 \\
Altitude range & $600-670 \mathrm{~m}$ \\
Turbine Heights & $49 \mathrm{~m}$ \\
Anemometric towers heights & $30 \mathrm{~m}$ \\
Vendor & Vestas \\
Model & $\mathrm{V} 52$ \\
$\mathrm{~N}^{\circ}$ blades & 3 \\
P peak & $850 \mathrm{~kW}$ \\
Rotor diameter & $52 \mathrm{~m}$ \\
Hub Height & $49 \mathrm{~m}$ \\
Cut-in wind speed & $4 \mathrm{~m} / \mathrm{s}$ \\
Cut-out wind speed & $25 \mathrm{~m} / \mathrm{s}$ \\
\hline
\end{tabular}

Figure 1 presents the layout (with red marks for the anemometric towers) of the proposed power plant and a photograph of some wind turbines. Most of the wind turbines were positioned cross the north direction while some of them (VZ36, VZ35, VZ34 VZ33) were clustered in another part of the zone.
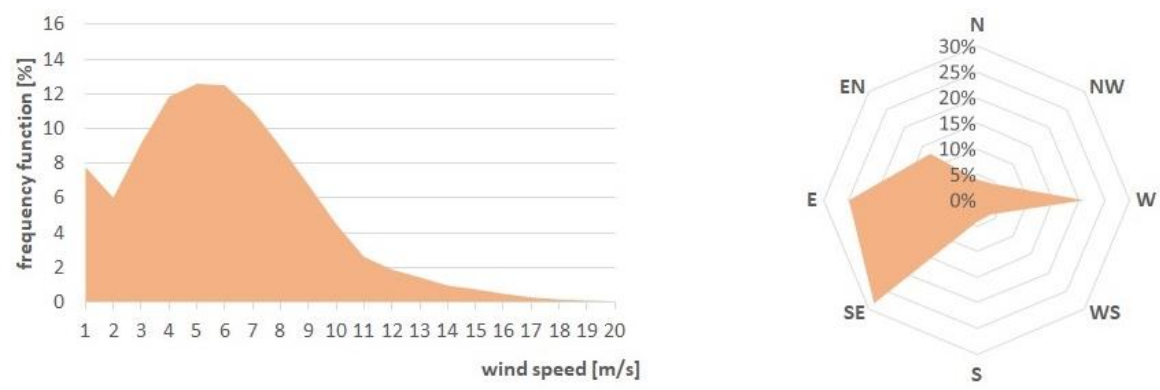

Fig. 2. The geographical layout of the wind turbines (a) and a photograph detail (b).

The range 4-7 [m/s] velocity is the most frequent, with a frequency of $36.13 \%$ in 2012 . It is more interesting to focus attention to the wind directions distribution. As consequence of right displacement wind turbines criteria, the predominant wind direction is aligned along the axis E-W (in both directions) with a frequency of $46.02 \%$ in 2012 . This is the most favorite scenario, when no power losses wind turbines, due to wake effects, occur. However, the axis NW-SE (especially from SE to NW), where most of the wind turbines are located, presents relevant frequency: $32.97 \%$. In this case most of the wind turbines were probably affected by wake phenomena causing significant power losses 


\section{Methods and Application}

In order to perform a medium-term forecast overall energy production it was implemented a methodology that combines a wake model, implemented with a GIS based approach and the use of a Multilayer perceptron (MLP) neural network.

\section{Wake mathematical model based on Jensen Theory}

There is a vast literature about wakes models for wind turbines in wind farm.

Among engineering models, The Jensen wake model is probably the most popular [12]. It was developed by Prof. Jensen in early 1980s and it has been widely used in many researchers $[11,13]$. It is a adapted to quantify the reduction of wind speeds downstream a wind turbine due to wakes phenomena. The almost real-time calculation of wind speeds losses beyond this approach derives from some assumptions on wakes propagation. In order to describe the wake derived from a specific wind turbines this model is based on the following simplifying assumptions:

- The wake expands linearly downstream the wind turbine;

- The velocity profile inside the wake is considered ideal and constant along the ray;

- The wake expands linearly in accordance with the decay coefficient;

- The aerodynamic characteristics of the turbines are described only by the thrust coefficient;

- The turbulence effects in the wake and in the wingtip vortices are not calculated.

The Momentum Conservation Principle around a control volume is the basis of this model applied to the turbine wake. The geometric evolution of the wake downstream a turbine follows Figure 3.
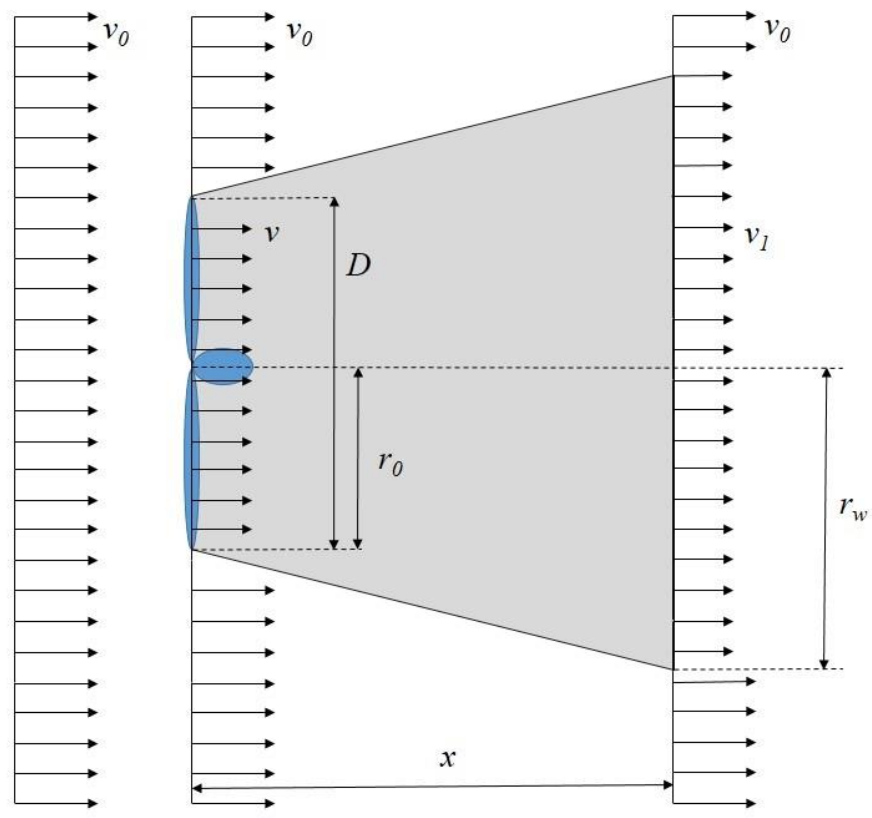

Fig. 3. A wake schematic representation according to Jensen theory. 
The Momentum Conservation Principle for a wind turbine is described as follow:

$$
\pi r_{0}^{2} v+\pi\left(r_{w}^{2}-r_{0}^{2}\right) v_{0}=\pi r_{w}^{2} v_{1}
$$

As illustrated in Figure 3,v is the velocity just behind the rotor, $v_{0}$ is the undisturbed velocity flow that is obviously grater in module than $v, v_{l}$ is the velocity downstream the rotor, $r_{0}$ is the rotor radius and $r_{w}$ is wake radius at a linear distance $x$ from the turbine. The velocity $v$ can be calculated by applying the Betz theory and it is a function of $v_{0}$ :

$$
v=(1-2 a) v_{0}
$$

Where a represents the axial induction coefficient. It depends on the geometry of the rotor. This coefficient in the original Jensen mathematical model, was set equal to $1 / 3$. However, as proposed in this paper, it more accurate to calculate it by considering the thrust coefficient $\left(\mathrm{C}_{-} \mathrm{T}\right)$, as reported in the following equation:

$$
a=\frac{\left(1-\sqrt{1-C_{T}}\right)}{2}
$$

The linear expansion of the wake is represented by the following equation, where $\alpha$ is the decay coefficient:

$$
r_{w}=(1+2 \alpha x) r_{0}
$$

The decay coefficient $(\alpha)$, that influences the dimension of the wake, is usually calculated taking into account the roughness. For the proposed case study the roughness was set to be 0.3 for all turbines since the landscape type refers to open agricultural areas without fences and hedgerows and very scattered buildings. The combination of Eq. (1), (2), (3) and (4) leads to velocity flow downstream the rotor $v_{l}$ that can be express in function of $C_{T}, \alpha$ and $x$ as follows:

$$
v_{1}=v_{0}\left[1-\frac{1-\sqrt{1-C_{T}}}{(1+2 \alpha x)^{2}}\right]
$$

In order to test this model in the proposed wind farm a linear sum of velocity deficits due to several turbines in the wake region, was assumed:

$$
v_{j}=v_{0}\left[1-\sqrt{\sum_{i=1}^{N}\left(1-\frac{v_{i}}{v_{0}}\right)}\right]
$$


Thanks to the eq. (6) the velocity $v$ at the turbine $j$ is calculated by considering the total number of upwind turbines $(N)$ which affect the target turbine $j$ by their wakes.

This mathematical model was adapted to build an overall energy production model applied to the case study.

First of all the 30 wind turbines and the 3 anemometric towers were georeferenced in a GIS-based system. The integration of spatial attributes such as geographical coordinates (Latitude and Longitude) and quotes of each point (turbines or towers) led to estimate relative distances among them and their different heights. Data from SCADA system for the reference year was collected. Data referred to real $\mathrm{P}_{\text {peak }}$ for each turbine, wind speeds and directions for each tower. A script was implemented in order to replace missing or wrong (negative powers e.g) values with a moving mean calculation by considering similar temporal subsets. However, this analysis referred to only the $1 \%$ of the total samples set. It was chosen to use the average values among anemometric towers data as reference undisturbed velocity flows $\left(v_{0}\right)$ directions $\left(w_{0}\right)$ and heights $\left(z_{r e f}\right)$. Other data were used for the following step such as: Thrust coefficient of the wind turbine (CT), power curve (as table), Rotor Diameter (D).

A spatial "fishnet" grid of squares in the field of the wind farm was created as reported in Figure 4(a). The dimensions of the squares were $2 D^{*} 2 \mathrm{D}$ so to not consider near-wake phenomena. This grid was converted to a binary table ( 1 value/turbine, 0 value/no turbine) of $110 * 110$ records with the row $\mathrm{i}$ representing the coordinate $\mathrm{y}$ and column $\mathrm{j}$ the coordinate $\mathrm{x}$ for each turbine in the reference system YOX. At each time step $\mathrm{h}$, the model handled the couple $\left(v_{0 h}, w_{0 h}\right)$ and created a new coordinates reference system ( $\left.Y^{\prime} \mathrm{O}^{\prime} \mathrm{X}^{\prime}\right)$ for each turbine with a rototranslation so to have the new origin $\left(\mathrm{O}^{\prime}\right)$ at the considered turbine position and axis $\mathrm{X}^{\prime}$ orientated along the same direction of $w_{0}$ with the opposite versus. The schematic representation of this transformation is described in Figure 4(b).

In this way, at each step, the other turbines that effect the considered turbine centered in $O$ ' with their wakes had to fulfill the following assumptions with their roto-translated coordinates $\left(x^{\prime}, y^{\prime}\right)$ :

$$
\left\{\begin{array}{c}
x^{\prime}>0 \\
\left|y^{\prime}\right| \leq r_{w}\left(x^{\prime}\right)
\end{array}\right.
$$

Where $\boldsymbol{r}_{\boldsymbol{w}}\left(\boldsymbol{x}^{\prime}\right)$ is the the wake ray calculated at the distance $x$ ' to the considered turbine in accordance to eq. 4 .

With these assumptions it was possible to estimate the income velocity flows at each turbine by considering eq. 5 and eq. 6 . Moreover, the model estimated the velocity deficit (vloss), due to wake phenomena, for each turbine $i$ at the time step $h$ as follows:

$$
v \operatorname{loss} s_{h}^{i}=\frac{v 0_{h}^{i}-v 1_{h}^{i}}{v 0_{h}^{i}}
$$

Where $v l$ and $v 0$ are respectively the income velocity flow and the undisturbed velocity flow for the turbine $i$ at the time step $h$. 

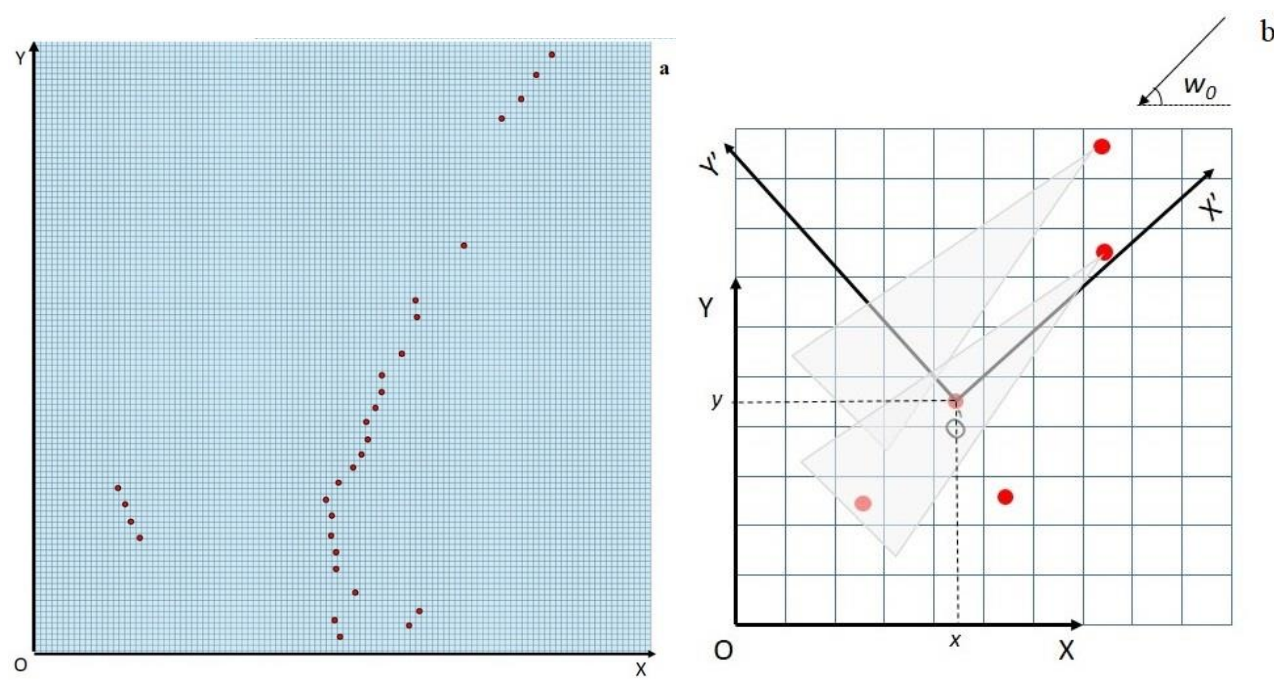

Fig. 4. The spatial fishnet net grid with turbines as red points (a) and the roto-translation (b) of the reference system $X O Y$ to $X^{\prime} O^{\prime} Y$ '.

\section{Forecast method with ANN}

In this paper, a Multilayer perceptron (MLP) neural network was used to conduct a medium-term overall wind power forecast (about seven days) in advance. This common neural network has been widely used in similar researchers [14,15]. MLP can give a reasonable solution for such a highly nonlinear problem as wind power forecast. It uses a feedforward architecture. It was modeled with three layers of nodes: input layer, hidden layer and an output layer as shown in Figure 5. Except for the input nodes, each node is a neuron that uses a nonlinear activation function. The sigmoid function was used As activation function which is probably the most historically common one. MLP uses a supervised learning technique called backpropagation for training. The Data subset was divided in two categories: the $70 \%$ was used for the learning phase and the $30 \%$ as test phase. MLP received as input the wind speeds and wind directions of three different turbines and gave as output the Overall Energy Production of the wind farm. 


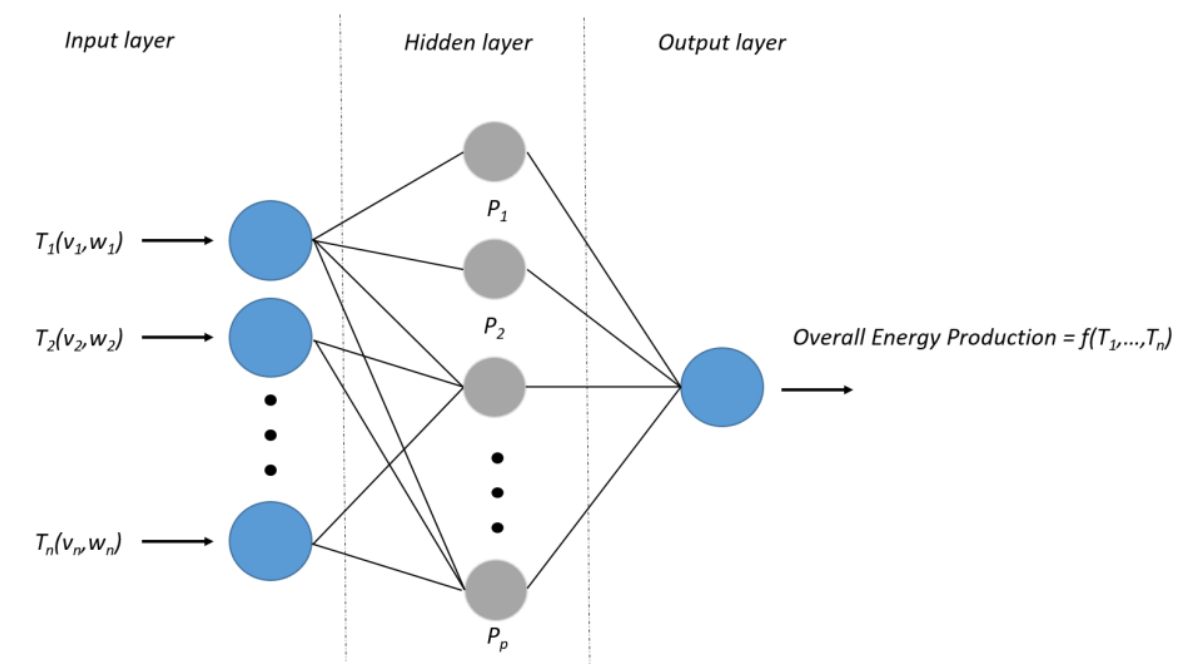

Fig. 5. The architecture of the Multilayer perceptron neural network adopted in this paper.

The model considered for each simulation those turbines that presented the minimum values of vloss for the reference period of simulation. It implied that the other turbines were affected more of wakes phenomena and so be "physically" correlated. Moreover, the results were compared with other approaches such as energy production based on Jensen estimation, the same MLP approach with random turbines as input and the same MLP approach with all turbines as input.

\section{Results}

As reported in the previous chapter, the neural network was tested for the year 2012 with a time step of one hour and one week forecast period. Model output were compared with observed data. Figure 6 shows results of the model for the $12^{\text {th }}$ week. It was deliberating decided to show only this week as it presents a typical trend if compared to the other weeks of 2012. The model follows observed data for almost all samples but some outliers. The trend, as shown in the figure, is almost linear demonstrating the promising results of this approach. Figure 7 presents more specific details of the same considered week in terms of relative errors distribution. Most of relative errors with observed data were less than $11 \%$ as reported with the frequencies in the figure. The average was $10.29 \%$ while the median value was $9.44 \%$. 


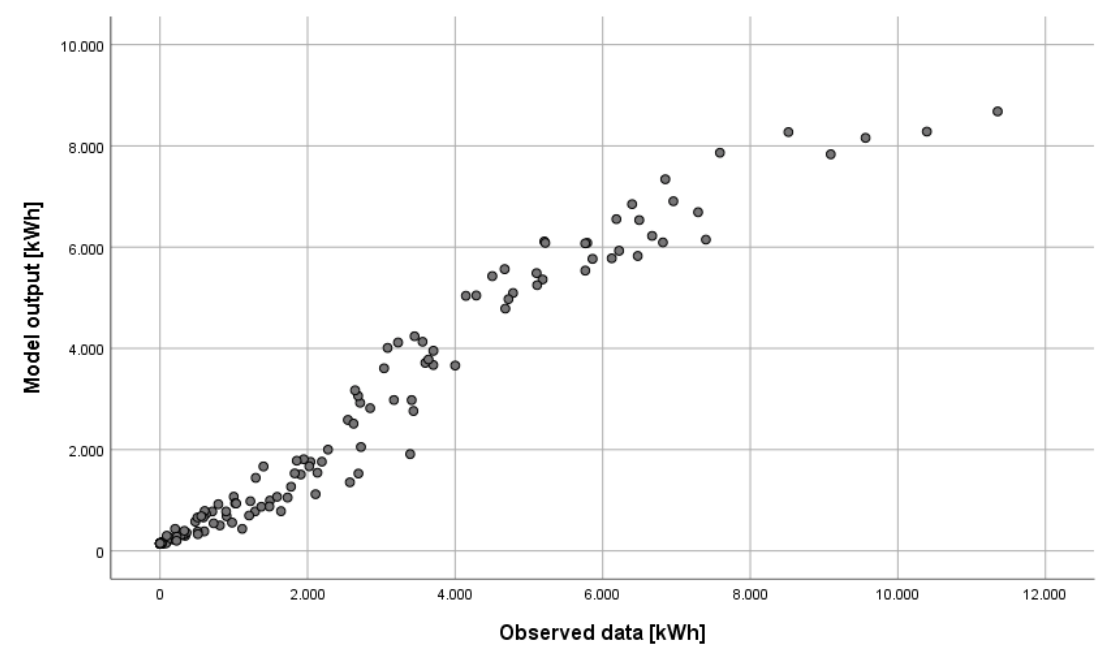

Fig. 6. The MLP model results of the overall energy production for the week 12 of 2012.

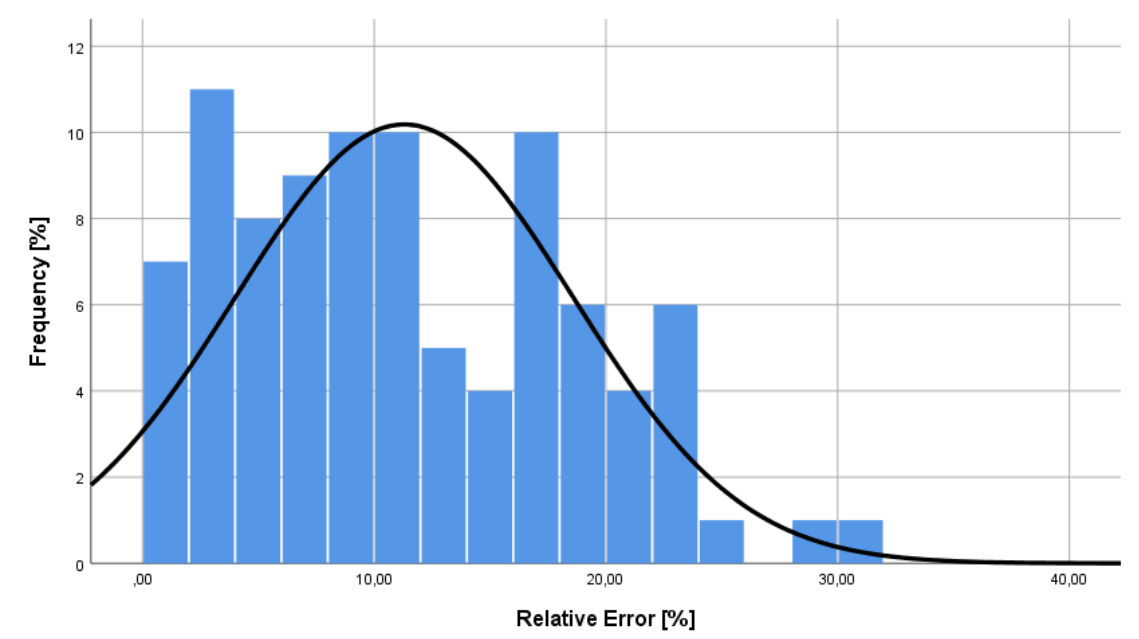

Fig. 7. The distribution of the relative error for the MLP model application in the week 12 of 2012.

The medium-term Overall Energy Production forecast results for the $12^{\text {th }}$ week of 2012 is reported in Figure 8. Results are reported in different scenario configurations.

The energy production has been calculated with 4 different approaches and compared with observed data: Jensen model application (Jensen), the application of MLP with indications from Jensen model as reported in the previous chapter (MLP + Jensen), the application of MLP with random turbines, the application of MLP with all turbines. Figure 8 shows as the proposed methodology (MLP + Jensen) presents best results. Moreover, it presents better results if compared with the applications of MLP with all turbines. This is probably caused by the phenomenon of overfitting since the number of parameters $(30$ turbines) is too high and the consequent structure is too complicated. 


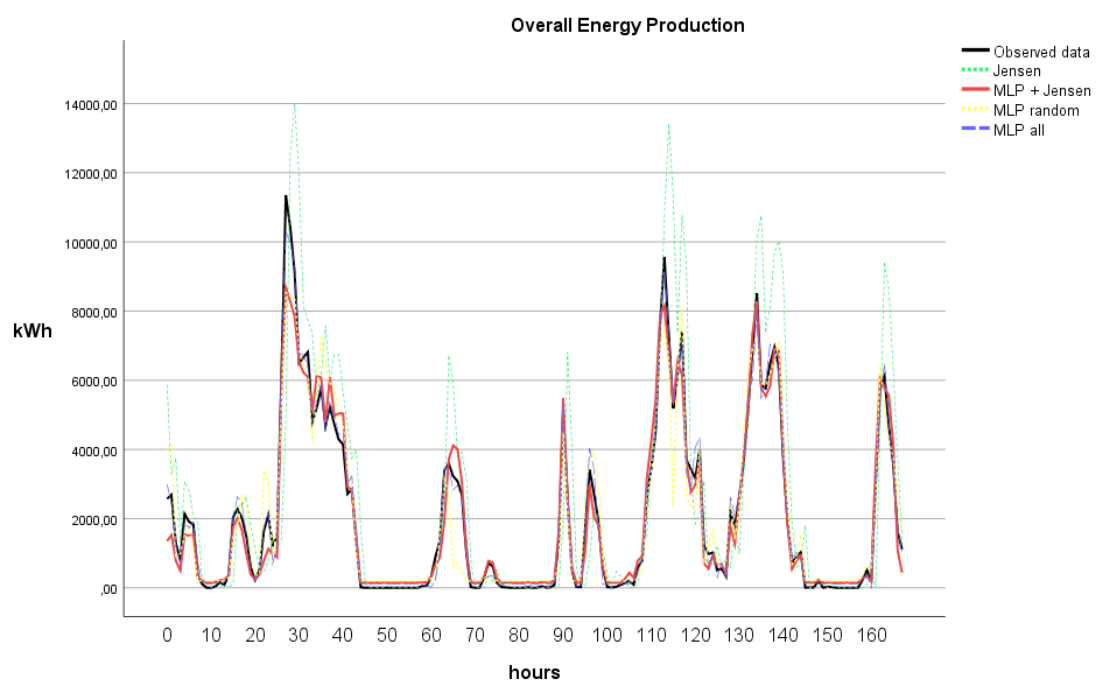

Fig. 8. The real and forecast energy production the week 12 of 2012.

\section{Conclusions}

This paper deals with a new methodology application in a medium-term overall energy production forecast in wind farms. This methodology consisted in the application of a wake model, implemented with a GIS based approach and the use of a Multilayer perceptron (MLP) neural network. The main goals reached can be summarized as follows:

- The proposed wake model, implemented with a GIS based approach, was effective in calculated wakes phenomena that followed observed data as reported in the case study.

- The application of MLP neural network for the medium-term energy production forecast led to robust results for all scenarios.

- The results of the model (Jensen application and MPL) improved considerably the accuracy of the medium-term overall energy production forecast. The application of this lower number of turbines was almost the same of the application of all turbines. This latter case usually suffer a heavy computation load not really adapted for near-real time applications such this one and may lead to overfitted results.

For future work it is under investigation the integration of other variables such as: slopes, terrain roughness, quotes, directions of all turbines. All these gis-based variables should lead to better performances for the model itself, especially for the characterization of wake effects. 


\section{Acknowledgements}

The research is supported by "programma ricerca di ateneo UNICT 2020--22 linea 2 " of the University of Catania.

\section{References}

1. “The World Wind Energy Report 2018”. World Wind Energy Agency (WWEA), (2019).

2. F. Chiacchio, D. D’Urso, F. Famoso, S. Brusca, J.I. Aizpurua, V. M. Catterson. "On the use of dynamic reliability for an accurate modelling of renewable power plants" in Energy, 151, 605-621 (2018).

3. F. Famoso, M. Prestipino, S. Brusca, A. Galvagno. "Designing sustainable bioenergy from residual biomass: Site allocation criteria and energy/exergy performance indicators" in Applied Energy, 274, 115315 (2020).

4. A. Galvagno, M. Prestipino, S. Maisano, F. Urbani, V. Chiodo. "Integration into a citrus juice factory of air-steam gasification and CHP system: Energy sustainability assessment" in Energy Conversion and Management, 193, (2019).

5. W. Gądek, M. Mlonka-Mędrala, M. Prestipino, P. Evangelopoulos, S. Kalisz, W. Yang. "Gasification and pyrolysis of different biomasses in lab scale system: a comparative study" in E3S Web Conf, 10, 00024, (2016).

6. S. Brusca, F. Famoso, A. Galvagno, R. Lanzafame, S. Mauro, M. Messina. "Wind turbine wake mathematical models validation by means of wind field data" in International Journal of Applied Engineering Research, 12(24), 16068-16076, (2017).

7. S. Brusca, F. Famoso, R. Lanzafame, A. Galvagno, S. Mauro, M. Messina. "Wind farm power forecasting: New algorithms with simplified mathematical structure" in AIP Conference Proceeding, 2191, 020028, (2019).

8. R. G. Kavasseri, K. Seetharaman. "Day-ahead wind speed forecasting using fARIMA models" in Renewable Energy, 34(5), 1388-1393, (2009).

9. L.A Landberg. "Mathematical look at physical power prediction model" in Wind Energy, 1-23, (1998).

10. D. Elia. "Wind turbine control in computational fluid dynamics with OpenFOAM" in Wind Engineering, 41(4), (2017).

11. S. Brusca, R. Lanzafame, F. Famoso, A. Galvagno, M. Messina, S. Mauro, M. Prestipino. "On the Wind Turbine Wake Mathematical Modelling" in Energy Procedia, 148, 202-209, (2018).

12. N. O. Jensen. "A note on wind generator interaction" in Technical Report Risø-M2411(EN), Risø National Laboratory, Roskilde, (1983).

13. L. Tian, W. Zhu, W. Shen, Y. Song, N. Zhao. "Prediction of multi-wake problems using an improved Jensen wake model" in Renewable Energy, 102, 457-469, (2017).

14. G.W. Chang, H.J. Lu, Y.R. Chang and Y.D. Lee. "An improved neural networkbased approach for short-term wind speed and power forecast" in Renewable Energy, 105, 301-311, (2017).

15. M. Carolin Mabel, E. Fernandez. "Analysis of wind power gneration and prediction using ANN: A case study” in Renewable Energy, 33(5), 986-992, (2008). 